\title{
Novel bi-allelic MSH4 variants causes meiotic arrest and non-obstructive azoospermia
}

\author{
Peng $\mathrm{Li}^{1 \dagger}$, Zhiyong $\mathrm{Ji}^{1}{ }^{1+}{ }^{+}$, Erlei Zhi ${ }^{1}$, Yuxiang Zhang ${ }^{1}$, Sha Han ${ }^{1}$, Liangyu Zhao ${ }^{1}$, Ruhui Tian ${ }^{1}$, Huixing Chen ${ }^{1}$,
} Yuhua Huang ${ }^{1}$, Jing Zhang ${ }^{3}$, Huirong Chen ${ }^{1}$, Fujun Zhao ${ }^{1}$, Zhi Zhou ${ }^{4^{*}}$, Zheng $\mathrm{Li}^{1^{*}}$ and Chencheng Yao ${ }^{1,4^{*}}$

\begin{abstract}
Background: Non-obstructive azoospermia (NOA) is one of the most severe type in male infertility, and the genetic causes of NOA with meiotic arrest remain elusive.

Methods: Four Chinese families with NOA participated in the study. We performed whole-exome sequencing (WES) for the four NOA-affected patients in four pedigrees. The candidate causative gene was further verified by Sanger sequencing. Hematoxylin and eosin staining (H\&E) and immunohistochemistry (IHC) were carried out to evaluate the stage of spermatogenesis arrested in the patients with NOA.

Results: We identified two novel homozygous frameshift mutations of MSH4 and two novel compound heterozygous variants in MSH4 in four pedigrees with NOA. Homozygous loss of function (LoF) variants in MSH4 was identified in the NOA-affected patient (P9359) in a consanguineous Chinese family (NM_002440.4: c.805_812del: p.V269Qfs*15) and one patient with NOA (P21504) in another Chinese family (NM_002440.4: c.2220_2223del:p.K741Rfs*2). Also, compound heterozygous variants in MSH4 were identified in two NOA-affected siblings (P9517 and P9517B) (NM_002440.4: c.G1950A: p.W650X and c.2179delG: p.D727Mfs*11), and the patient with NOA (P9540) (NM_002440.4: c.G244A: p.G82S and c.670delT: p.L224Cfs*3). Histological analysis demonstrated lack of spermatozoa in seminiferous tubules of all patients and $\mathrm{IHC}$ showed the spermatogenesis arrested at the meiotic prophase I stage. Consistent with the autosomal recessive mode of inheritance, all of these mutations were inherited from heterozygous parental carriers.
\end{abstract}

Conclusions: We identified that six novel mutations in MSH4 responsible for meiotic arrest and NOA. And these results provide researchers with a new insight to understand the genetic etiology of NOA and to identify new loci for genetic counselling of NOA.

Keywords: Non-obstructive azoospermia, Meiosis, MSH4, Male infertility

*Correspondence: zhouzhi@shanghaitech.edu.cn; lizhengboshi@sjtu.edu.cn; yaochencheng@126.com

${ }^{\dagger}$ Peng Li and Zhiyong Ji contributed equally to this work.

1 Department of Andrology, Center for Men's Health, Department of ART, Institute of Urology, Urologic Medical Center, Shanghai General Hospital, Shanghai Jiao Tong University School of Medicine, Shanghai 200080 China

${ }^{4}$ School of Life Science and Technology, ShanghaiTech University, Shanghai 201210, China

Full list of author information is available at the end of the article

\section{Introduction}

Male infertility is a multifactorial heterogeneous pathological condition, affecting $\sim 7 \%$ of male general population. Non-obstructive azoospermia (NOA) is one of the most severe male reproductive diseases, which occurs in $\sim 1 \%$ of men [1]. NOA displays germ cell absence or reduction owing to the testicular atrophy, and NOA usually results from $\mathrm{Y}$ chromosome microdeletion, chromosome abnormalities, hypogonadism, varicocele, testicular tumor, and improper drug administration. However, 70\% original author(s) and the source, provide a link to the Creative Commons licence, and indicate if changes were made. The images or other third party material in this article are included in the article's Creative Commons licence, unless indicated otherwise in a credit line to the material. If material is not included in the article's Creative Commons licence and your intended use is not permitted by statutory regulation or exceeds the permitted use, you will need to obtain permission directly from the copyright holder. To view a copy of this licence, visit http://creativecommons.org/licenses/by/4.0/. The Creative Commons Public Domain Dedication waiver (http://creativeco mmons.org/publicdomain/zero/1.0/) applies to the data made available in this article, unless otherwise stated in a credit line to the data. 
of patients with NOA were idiopathic, the etiology and pathology of NOA remains unclear.

Meiosis is an intricate process during the spermatogenesis, including one round of DNA replication and two rounds of chromosome segregation. The defect of meiosis could lead to meiotic arrest and NOA. During the meiosis prophase I, homologous recombination resulted in crossover (CO) formation in the context of the synaptonemal complex (SC). It's initiated by formation of DNA double-strand breaks via catalyzation of SPO11 [2]. And the recombination intermediates are repaired towards either $\mathrm{CO}$ or non-crossover (NCO) pathways [3]. The heterodimer of MutL (MLH1 and MLH3) is responsible for formation of the major COs [47], and MutS functions to recruit MutL to the SC during pachynema. Unlike MSH2-MSH6 (MutS $\alpha$ ) and MSH2MSH3 (MutS $\beta$ ) heterodimers, which are involved in mismatch repair during mitosis, MSH4-MSH5 (MutSy) functions exclusively during meiotic prophase I [8]. It has been demonstrated that loss of Msh4 or Msh5 results in defects of prophase I progression in mice, with almost complete failure of homologous synapsis, and cell death prior to pachynema [9-11]. Also, up to now, wholeexome sequencing (WES) of pedigree studies reported that only two types of homozygous mutation in $\mathrm{MSH} 4$ (NM_002440.4: c.2261C > T and c.1552C > T) were associated with NOA $[12,13]$. Thus, many other types of mutation in MSH4 which were associated with NOA remain to be elucidated.

Here, we identified six novel mutations in the meiotic gene MSH4 in four pedigrees with NOA. Two homozygous frameshift mutations were identified in two families. Also, two compound heterozygous mutations in MSH4 were reported in two other pedigrees with NOA.

\section{Materials and methods Study approval}

This study was approved by the Institutional Ethical Review Committee of Shanghai General Hospital, Shanghai Jiao Tong University (2020SQ199), and the informed consent of clinical data and testicular tissue for research was obtained from the donors.

\section{Study subjects}

Four families with NOA were enrolled in this study, and all the NOA-affected patients had no known causal factors for male infertility, including varicocele, radiation, chemotherapy, orchitis, cryptorchidism, and testicular cancer. The family histories were collected. Semen analysis and sexual hormones examination were performed in all the NOA-affected patients. Also, the genetic evaluation including karyotype, Y chromosome microdeletions, and WES were conducted in all the patients with NOA. Furthermore, routine testicular biopsy was performed for histopathological examination.

\section{WES}

The genomic DNA was extracted from the blood of the four probands using the TIANamp Blood DNA Kit (TIANGEN) according to the manufacturer's instructions. DNA was fragmented through Covaris focused ultrasonication. Known exons and exon-intron boundary sequences were captured using $\mathrm{xGen}^{\circledR}$ Exome Research Panel (IDT, USA), and sequencing DNA libraries were prepared following the manufacturer's instruction. Sequencing was performed on an Illumina HiSeq $\times 10$ platform. Sequencing reads were aligned to the human genome (GRCh37/hg19) using Burrows-Wheeler Aligner. Both single-nucleotide variants and indels within the captured coding exonic intervals were called using GATK, Platypus, VarScan, LoFreq, FreeBayes, SNVer, SAMtools and VarDict. Also, the variants were filtered and annotated using the ANNOVAR software. Because autosomal recessive or $\mathrm{X}$-linked inheritance were assumed for meiotic defect, genes with two alleles of potentially deleterious missense mutations (SIFT, PolyPhen-2 and Mutation Taster), or LoF mutations were kept for further analysis. Moreover, we compared candidate genes with human germline-enriched genes in the database and known pathogenic genes for azoospermia in mice. The aforementioned sequencing and bioinformatic analyses were conducted together with the Nuprobe company (Shanghai, China). The datasets used and analyzed during the current study are available from the corresponding author on reasonable request.

\section{Sanger sequencing}

Polymerase chain reaction (PCR) was performed in four families with NOA. The primers were shown in supplementary Table S1. And the PCR products were bidirectionally sequenced by Sanger sequencing using a 3730xl DNA Analyzer (Applied Biosystems, Forster City, California, USA).

\section{H\&E staining}

The testicular tissues from the NOA-affected probands were fixed in $4 \%$ paraformaldehyde solution overnight, embedded in paraffin and sectioned at $5 \mu \mathrm{m}$ thickness. Paraffin sections were dewaxed with xylene for $30 \mathrm{~min}$, and then, rehydrated sequentially in $95,90,85$ and $70 \%$ ethanol each for $10 \mathrm{~min}$, followed in PBS solution for $10 \mathrm{~min}$, and stained with hematoxylin and eosin according to standard protocols (catalogue number: ab245880, Abcam, Cambridge, UK). All the staining 
sections were captured by phase-contrast microscope to observe the structure changes of testicular tissues (Leica).

\section{IHC}

The testicular biopsies were obtained from the four patients with NOA and the OA-affected patient as the positive control. The testicular tissue was fixed overnight in $4 \%$ paraformaldehyde at $4{ }^{\circ} \mathrm{C}$, and then embedded in warm paraffin $\left(60^{\circ} \mathrm{C}\right)$. The biopsies were sectioned at $5 \mu \mathrm{m}$ thickness. The tissue sections were dewaxed in xylene, re-hydrated in a descending alcohol gradient, and heated in sodium citrate buffer $\left(90-98^{\circ} \mathrm{C}\right)$ for $15 \mathrm{~min}$ for antigen retrieval. After blocking with $5 \%$ BSA for $1 \mathrm{~h}$ at room temperature, the sections were incubated overnight with anti-SYCP3 (dilution: 1:25; catalogue number: AF3750, R\&D Systems), anti- $\gamma \mathrm{H} 2 \mathrm{AX}$ (dilution: 1:300; catalogue number: 2668445, Millipore), antiDMC1 (dilution: 1:100; catalogue number: sc-373,862, Santa Cruz) and PNA (dilution: 1:400; catalogue number: L21409, Thermo Fisher Scientific) at $4{ }^{\circ} \mathrm{C}$. The sections were washed thrice with PBS-T (Phosphate buffer saline-Tween), and incubated with highly cross-adsorbed secondary antibody conjugated with Alexa Fluor ${ }^{\circledR} 488$ or Alexa Fluor ${ }^{\circledR} 594$ (dilution: 1:400; Thermo Fisher Scientific) for $1 \mathrm{~h}$ at room temperature. The sections were washed three times with PBS-T and counterstained with 4',6-diamidino-2-phenylindole to label the nuclei. The images were captured by fluorescence microscope (Leica).

\section{Results}

\section{Clinical findings}

Four Chinese families with infertility participated in the present study (Fig. 1). All the probands had a history of male infertility for several years, and routine semen analyses revealed complete azoospermia with normal volume. They were in good physical condition. The testes were palpable and bilateral testicular size was normal, without varicocele. Laboratory examination showed that sex hormone levels in the four patients were comparable to the reference values. They had normal 46, XY karyotypes and no Y chromosome microdeletions. In family P9540, the parents of the proband were married first cousins, however, there was no family histories of consanguinity in other three families. The probands in four families underwent the testicular sperm extraction procedure in Shanghai General Hospital, and histopathological analysis revealed meiotic arrest in the four patients with NOA. Collectively, all the clinical characteristics of the NOA-affected patients were summarized in Table 1.

\section{Identification of the $\mathrm{MSH} 4$ mutations in four pedigrees with NOA}

WES was performed on the probands in the four families. After the genetic analyses pipeline, homozygous LoF variant in MSH4 (family P9359 (NM_002440.4: c.805_812del:p.V269Qfs*15); family P21504 (NM_002440.4: c.2220_2223del:p.K741Rfs*2), and compound heterozygous mutations in MSH4 (P9517 (NM_002440.4: c.G1950A:p.W650X and c.2179delG:p. D727Mfs*11; P9540 (NM_002440.4: c.G244A:p.G82S and c.670delT:p.L224Cfs*3)) were assumed as the most likely causes of meiosis defect in the four patients with NOA, respectively (Table 2).

Sanger sequencing was then performed to validate the results in WES sequencing using DNA samples from the family members. In family P9359, homozygous LoF mutation in MSH4 (NM_002440.4: c.805_812del:p. V269Qfs"15) was identified in the proband (Fig. 1A, B), and this frameshift variant resulted in a truncated MSH4 protein without expression of MutS III-V domain. In family P21504, homozygous LoF mutations in MSH4 (NM_002440.4: c.2220_2223del:p.K741Rfs*2) also led to a truncated MSH4 protein with the defect in expression of MutS V domain. Consistent with an autosomal recessive mode of inheritance, the unaffected parents were heterozygous carriers of this same $\mathrm{MSH} 4$ variant (Fig. 1G, H). In family P9517, compound heterozygous LoF mutations in MSH4 (NM_002440.4: c.G1950A:p. W650X and c.2179delG: p.D727Mfs*11) were identified in the proband and his brother (Fig. 1C, D). A maternally inherited nonsense variant (NM_002440.4: c.G1950A:p. W650X) resulted in a premature stop, and a paternally inherited frameshift variant (NM_002440.4: c.2179delG: p.D727Mfs*11) led to a truncated MSH4 protein. These two variants resulted in the defect in expression of MutS $\mathrm{V}$ domain. Furthermore, the proband's sister, who carried heterozygous paternal inherited frameshift variant (NM_002440.4: c.2179delG:p.D727Mfs"11) has two daughters, suggesting that compound heterozygous LoF mutations in MSH4 were the cause of NOA in the two brothers. In family P9540, a maternally inherited frameshift variant (NM_002440.4: c.670delT:p. L224Cfs*3) led to a truncated MSH4 protein without expression of MutS III-V domain and a paternally inherited variant (NM_002440.4: c.G244A: p.G82S) resulted in transition of Glycine to Serine at the position 82 of MSH4. And it showed strong evolutionary conservation at this position, and this missense variant was predicted to be potentially deleterious simultaneously by SIFT and MutationTaster. Altogether, two homozygous LoF mutations and two compound heterozygous mutations in MSH4 were identified in four patients with NOA (Fig. 2). 


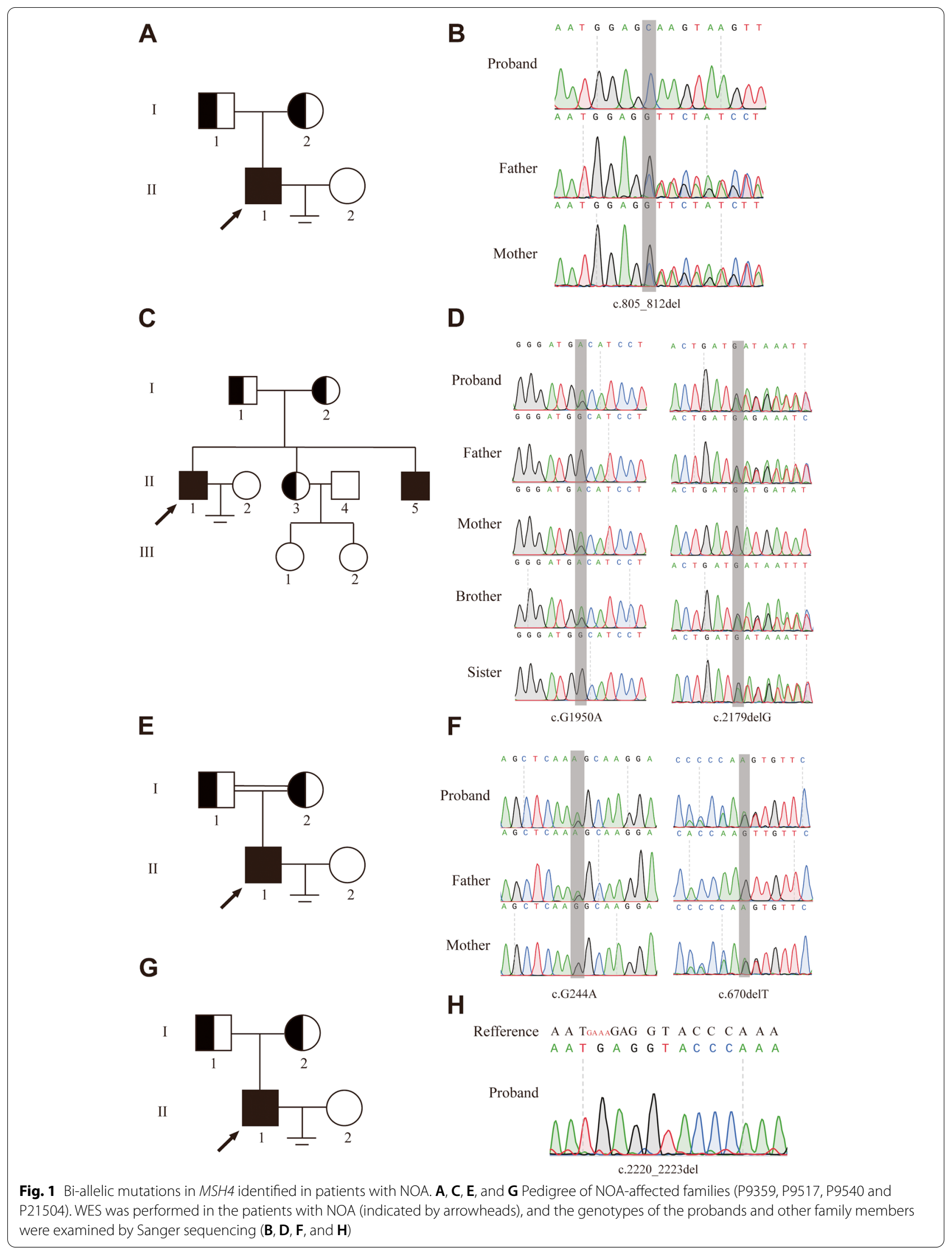


Table 1 Clinical characteristics of the patients with bi-allelic variants of MSH4 at the clinical assessment

\begin{tabular}{|c|c|c|c|c|c|}
\hline Subject & P9359 & P9517-1 & P9517-2 & P9540 & P21504 \\
\hline Secondary sexual characteristics & Normal & Normal & Normal & Normal & Normal \\
\hline Testicular volume (Left/Right, ml) & $12 / 12$ & $12 / 12$ & / & $12 / 12$ & $15 / 15$ \\
\hline Karyotype & $46, X Y$ & $46, X Y$ & / & $46, X Y$ & $46, X Y$ \\
\hline Y Chromosome microdeletions & No & No & / & No & No \\
\hline Follicle-stimulating hormone (IU/L) & 4.4 & 9.66 & / & 10.83 & 4.7 \\
\hline Luteinizing hormone (IU/L) & 5.54 & 5.52 & / & 5.44 & 3.4 \\
\hline Testosterone (nmol/L) & 3.45 & 3.95 & / & 5.63 & 2.87 \\
\hline
\end{tabular}

Table 2 In silico analysis of the novel variants of MSH4

\begin{tabular}{|c|c|c|c|c|c|c|c|c|c|}
\hline Patient No. & $\begin{array}{l}\text { Change in } \\
\text { coding DNA } \\
\text { (NM_001003811) }\end{array}$ & $\begin{array}{l}\text { Protein/RNA } \\
\text { change }\end{array}$ & Phenotype & $\begin{array}{l}\text { GnomAD } \\
\text { minor allele } \\
\text { frequency (\%) }\end{array}$ & ExAC & Polyphen2 & SIFT & $\begin{array}{l}\text { Mutation } \\
\text { taster }\end{array}$ & CADD \\
\hline P9359 & c.805_812del & p.V269Qfs*15 & MA & NA & NA & NA & NA & NA & NA \\
\hline P9517 & c.G1950A & p.W650X & MA & $1.2 \times 10^{-5}$ & $8.3 \times 10^{-6}$ & NA & NA & $\begin{array}{l}\text { disease_caus- } \\
\text { ing_automatic }\end{array}$ & NA \\
\hline P9517 & c.2179delG & p.D727Mfs*11 & MA & NA & NA & NA & NA & NA & NA \\
\hline P9540 & c.G244A & p.G82S & MA & NA & NA & Benign & Deleterious & Deleterious & NA \\
\hline P9540 & c.670delT & p.L224Cfs*3 & MA & NA & NA & NA & NA & NA & NA \\
\hline P21504 & c.2220_2223del & p.K741Rfs*2 & MA & NA & NA & NA & NA & NA & NA \\
\hline
\end{tabular}

Abbreviations: MA Maturation Arrest, NA Not Available






\section{Meiotic arrest phenotype in the patients with bi-allelic mutations in $\mathrm{MSH} 4$}

Four patients (P9517, P9540, P9359, P21504) received testicular biopsies, and no sperm was found. Compared with the testis of OA-affected patients (the positive control), the testicular histology from NOA-affected patients showed decreased number of spermatocytes and absence of spermatozoa and spermatids, but the number of spermatogonia and Sertoli cells at the basement membrane within the tubules remained unchanged (Fig. 3). IHC revealed the expression of DMC1 (indication for doublestrand break repair) in the affected patients' seminiferous tubules. However, there was no signal of PNA (a signal of acrosome), which is a marker of spermatids and spermatozoa (Fig. 4A-D). Furthermore, there were positive expressions of SYCP3 and $\gamma \mathrm{H} 2 \mathrm{AX}$ signal in the NOAaffected patients (Fig. 5A-D). SYCP3 was used to label components of the axial/lateral element. In preleptotene to zygotene spermatocytes, $\gamma \mathrm{H} 2 \mathrm{AX}$ staining showed pan-nuclear signal, whereas it could also label XY body, a visibly distinct domain in the nucleus of the pachytene spermatocytes. In the four NOA-affected patients, $\gamma \mathrm{H} 2 \mathrm{AX}$ was prominent in the preleptotene to zygotene spermatocytes, however, XY body was not detected in the biopsy (Fig. 5A-D). In contrary, the expression of SYCP3, $\gamma \mathrm{H} 2 \mathrm{AX}, \mathrm{DMC} 1$ and PNA were positive in the

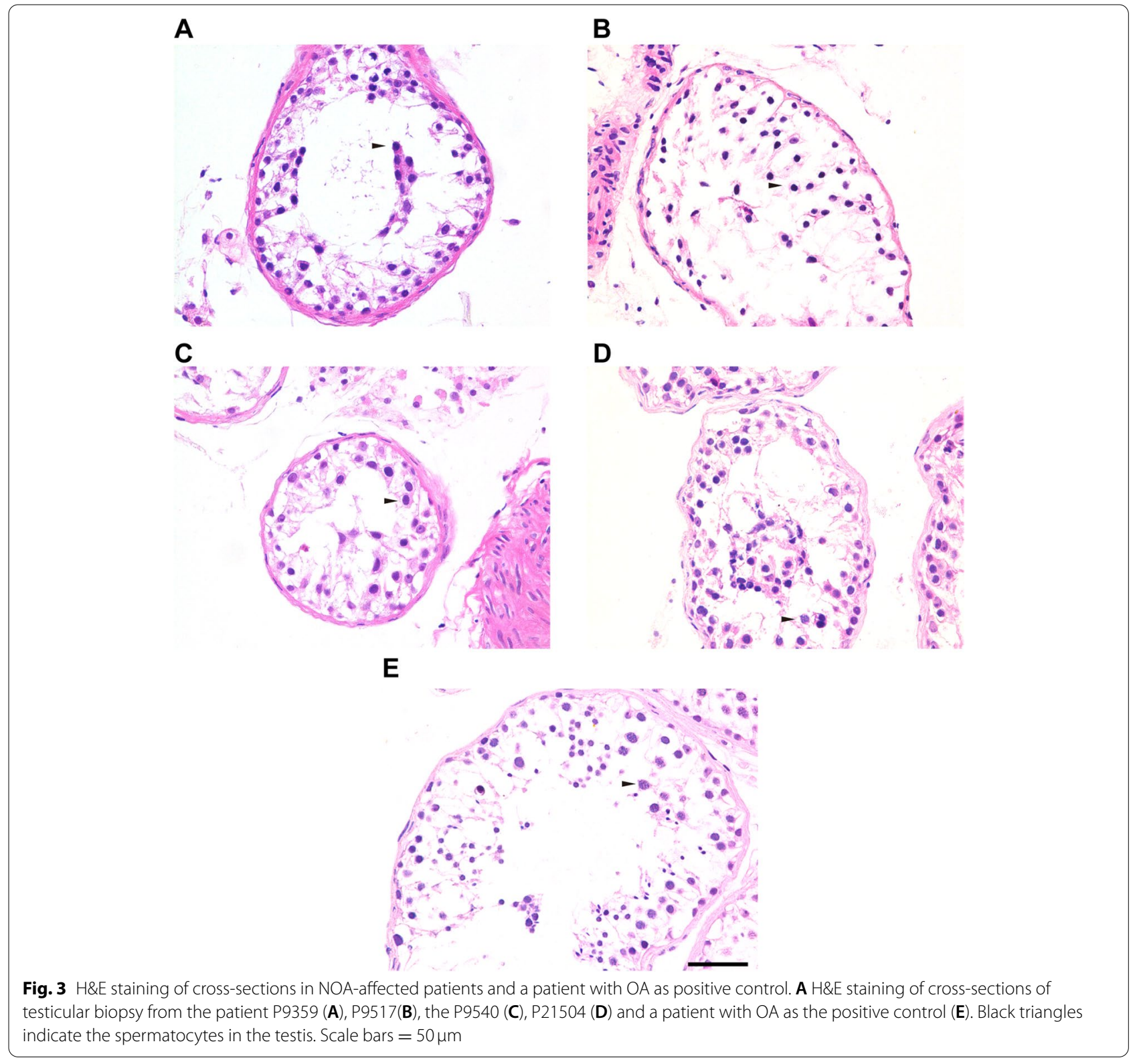



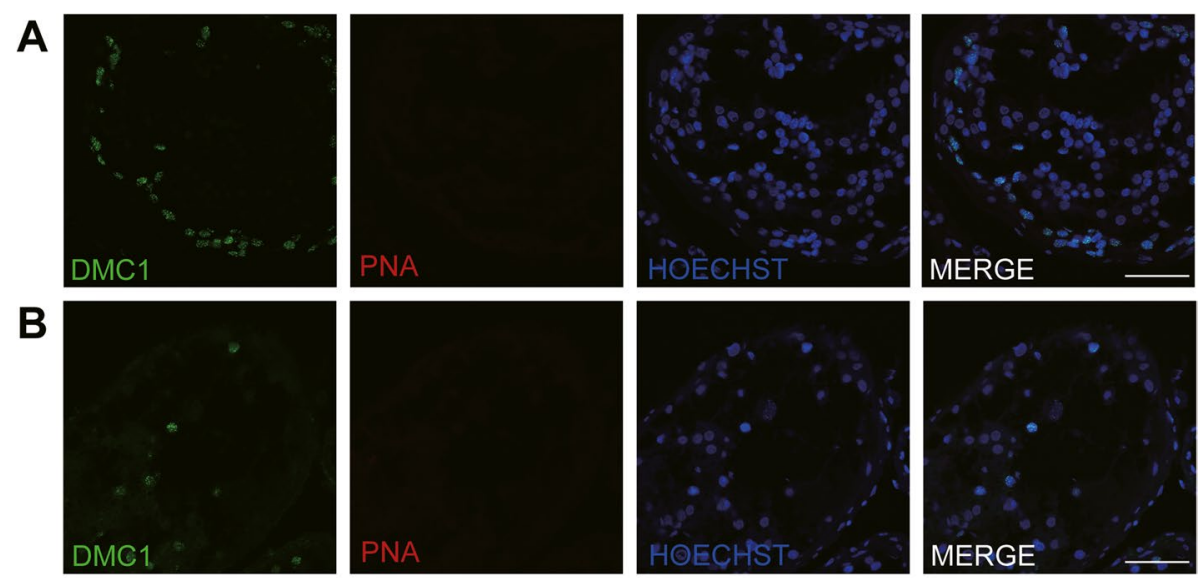

C
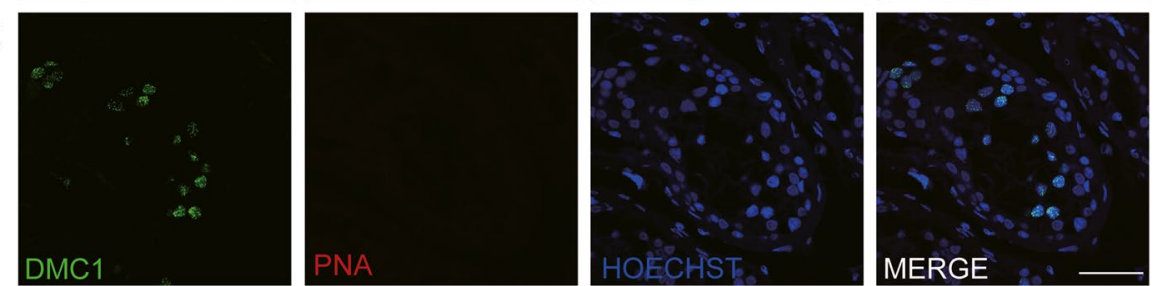

D


E
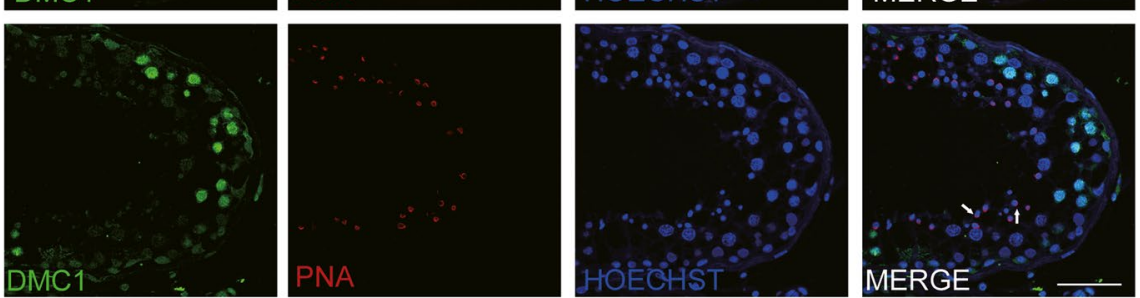

Fig. 4 Expression of recombination proteins DMC1 and acrosomal marker PNA in the testis of patients with NOA and a patient with OA as a positive control. IHC staining showed the expression of DMC1 (green) and PNA (red) in the testis of the P9359 (A), P9517 (B), P9540 (C), P21504 (D), and a patient with $\mathrm{OA}(\mathbf{E})$. Arrow indicates the acrosome of spermatids in the testis. Scale bars $=50 \mu \mathrm{m}$

OA's testis (Figs. 4E and 5E). Altogether, the results indicated spermatocytes stage arrest for all the four NOAaffected patients with bi-allelic mutations in $\mathrm{MSH}$.

\section{Discussion}

In the current study, six novel variants in $\mathrm{MSH} 4$ were identified in the four NOA-affected patients, including two homozygous variants and two compound heterozygous variants. In silico analysis and functional assays revealed the pathogenicity of the variants in $\mathrm{MSH} 4$, suggesting these 6 novel variants could cause meiotic arrest and NOA.
NOA can be classified into Sertoli cell only syndrome, maturation arrest (MA), and hypospermatogenesis [14]. Uniform MA is a unique subset of $\mathrm{NOA}$, and the incidence of genetic disorders in NOA patients with MA is higher than other types of NOA [15]. To date, several genes have been identified to be associated with meiosis defects in human via pedigree study, including TEX11(OMIM:300311), SHOC1 (OMIM:618038), TEX14 (OMIM: 605792), STAG3 (OMIM: 608489), DMC1 (OMIM: 602721), SYCE1 (OMIM: 611486), MSH5 (OMIM: 603382) and MSH4 (OMIM: 602105) [16-22]. 

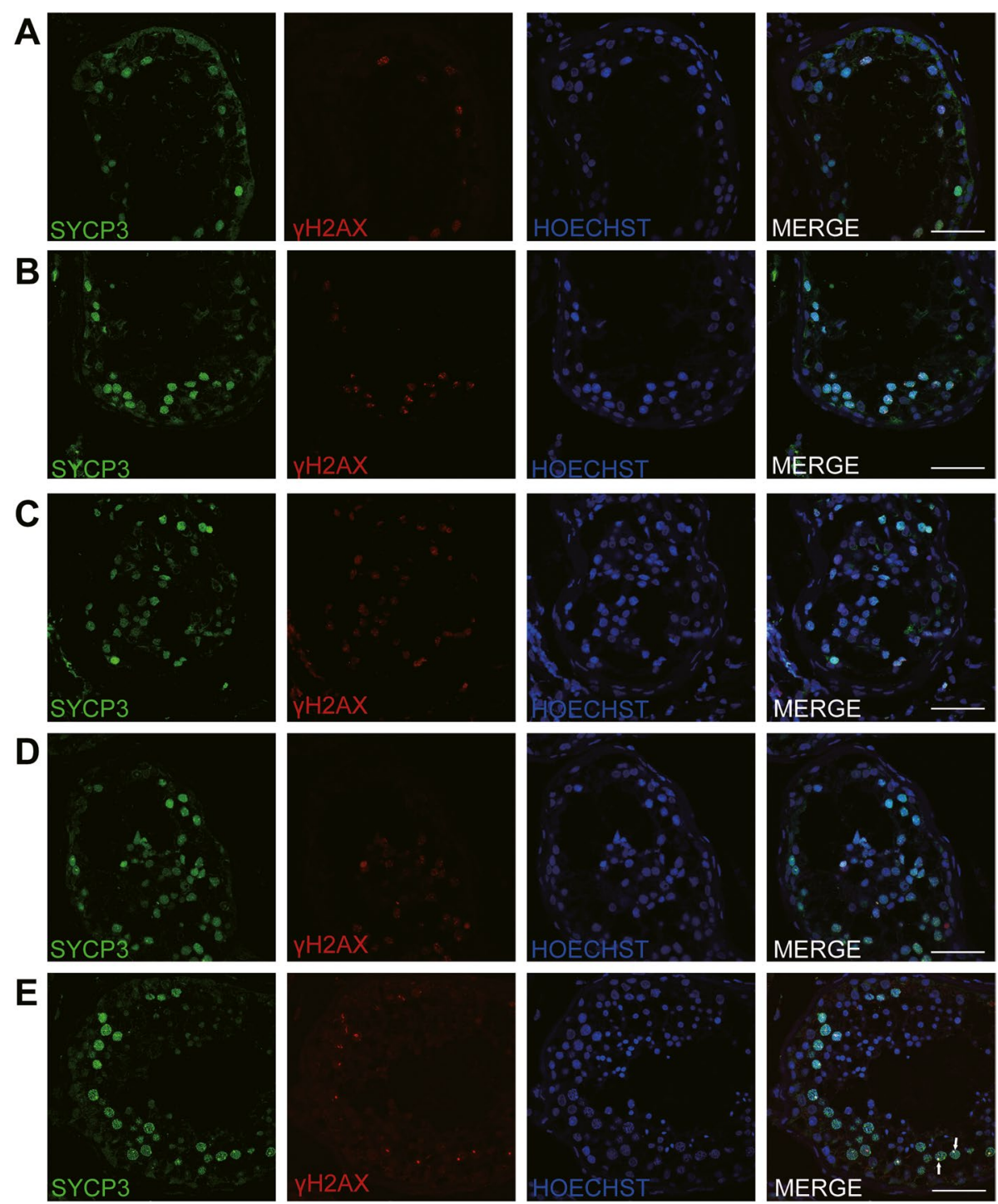

Fig. 5 Expression of SYCP3 and $\gamma H 2 A X$ in the testis of NOA-affected patients and a patient with OA as positive control. IHC staining showed the expression of SYCP3 (green) and YH2AX (red) in the testis of the P9359 (A), P9517 (B), P9540 (C), P21504 (D), and a patient with OA (E). The arrow indicates the $X Y$ body in the spermatocytes in the testis. Scale bars $=50 \mu \mathrm{m}$

MSH4, a meiosis-specific gene located at $1 \mathrm{p} 31.1$ in human, has 20 exons encoding a 936-aa protein. MSH4 belongs to the DNA mismatch repair family of proteins. And MSH4 could form an obligate heterodimer with MutS homolog partner MSH5, which is essential in meiotic recombination. The loss of Msh4 or Msh5 in mice resulted in defects of prophase I progression, with almost complete failure of homologous synapsis [9-11]. To date, WES of pedigree studies reported only two types of homozygous mutation in MSH4 (NM_002440.4: c. $2261 \mathrm{C}>\mathrm{T}$ and c.1552C $>\mathrm{T}$ ) were associated with NOA
$[12,13]$. MutS heterodimers are characterized by five distinct MutS domains (I-V), and each domain is responsible for a specific function. MutS domain I recognizes DNA mismatches, and MutS domain II functions as a connector between MutS domain I and MutS domain III (the core). Furthermore, MutS domain V (the ATPase domain) contains the $\mathrm{ABC}$ responsible for conformational changes in the clamp structure. Collectively, MSH4-MSH5 dimer is essential for meiotic recombination.

In the current study, we reported that six novel MSH4 variants were associated with meiotic arrest 
and NOA. The homozygous LoF mutations in MSH4 (NM_002440.4: c.805_812del:p.V269Qfs*15; and NM_002440.4: c.2220_2223del:p.K741Rfs*2) led to a truncated MSH4 protein without expression of the ATP-binding domain, which has been demonstrated to be essential for MSH4 function. The compound heterozygous LoF mutations in MSH4 (NM_002440.4: c.G1950A:p.W650X and c.2179delG: p.D727Mfs*11, respectively) were identified in family P9517. The allele frequency of maternal MSH4 variant NM_002440.4: c.G1950A:p.W650X (rs149910287) was $8.3 \times 10^{-6}$ in the ExAC_All database, while the allele frequency of paternal MSH4 variant NM_002440.4: c.2179delG:p.D727Mfs*11 has not been reported before (https://gnomad.broadinsti tute.org/). And these two variants resulted in the defect in expression of MutS V domain. In family P9540, a maternally inherited frameshift variant (NM_002440.4: c.670delT: p.L224Cfs*3) led to a truncated MSH4 protein without expression of MutS III-V domain and a paternally inherited variant (NM_002440.4: c.G244A: p.G82S) resulted in transition of Glycine to Serine at the position 82 of MSH4. And this missense variant was predicted to be potentially deleterious simultaneously by SIFT and MutationTaster.

In the current study, IHC facilitated to more accurately evaluate the period of spermatogenesis arrest. It was showed that DMC1 was expressed in all the testicular tissue with bi-allelic $M S H 4$ mutations, suggesting normal initiation of DSB repair in these patients. However, signals of PNA labeling spermatids and spermatozoa could not be detected in these biopsies. In normal spermatogenesis, $\gamma \mathrm{H} 2 \mathrm{AX}$ expression showed pan-nuclear pattern during preleptotene to zygotene stages, and in the pachytene stage, $\gamma \mathrm{H} 2 \mathrm{AX}$-foci represented sex bodies. SYCP3 is a marker of the axial/ lateral element (AE and LE). During the leptotene and zygotene, $\mathrm{AE}$ forms and extends, and in the pachytene stage, complete synapses form. However, there was no signals of $\mathrm{XY}$ body indicated by $\gamma \mathrm{H} 2 \mathrm{AX}$ staining in the seminiferous tubules of the patients with bi-allelic MSH4 mutations. And SYCP3 exhibited the characteristics of the zygotene stage, suggesting the spermatogenesis arrested at zygotene in these patients with NOA.

In conclusion, we identified six novel mutations in the meiotic gene MSH4 in four pedigrees with NOA. The meiotic arrest phenotype was confirmed in the NOA-affected patient. Further studies are required to identify the pathological role of the missense variant (NM_002440.4: c.G244A: p.G82S) and investigate the contribution of $\mathrm{MSH} 4$ variants in infertile men in larger cohorts.
Abbreviations

CO: Crossover; DSBs: Double-strand breaks; H\&E: Hematoxylin and eosin staining; IHC: Immunohistochemistry; LoF: Loss of function; MA: Maturation arrest; NCO: Non-crossover; NOA: Non-obstructive azoospermia; OA: Obstructive azoospermia; PCR: Polymerase chain reaction; SC: Synaptonemal complex; SIFT: Sorting Intolerant From Tolerant; WES: Whole-exome sequencing.

\section{Supplementary Information}

The online version contains supplementary material available at https://doi. org/10.1186/s12958-022-00900-x.

Additional file 1: Figure S1. The conservation of missense mutation (p.G82S) in MSH4 protein. The conserved glycine amino acid at position 82 was changed to serine amino acid.

Additional file 2: Table S1. Primer sequences used for Sanger sequencing.

\section{Acknowledgements}

We would like to thank all the individuals who participated in and supported this research.

\author{
Web resources \\ OMIM, https://www.omim.org/ \\ SIFT, http://sift.jcvi.org \\ ENCODE, https://www.encodeproject.org \\ Exome Aggregation Consortium (ExAC) Browser, https://gnomad.broad \\ institute.org/ \\ FANTOM, http://fantom.gsc.riken.jp \\ GenBank, https://www.ncbi.nlm.nih.gov/genbank/ \\ gnomAD, http://gnomad.broadinstitute.org \\ GTEx, http://www.gtexportal.org \\ PolyPhen-2, http://genetics.bwh.harvard.edu/pph2 \\ dbSNP, https://www.ncbi.nlm.nih.gov/projects/SNP/ \\ MGl, http://www.informatics.jax.org/mgihome/homepages/ \\ Human Protein Atlas, https://www.proteinatlas.org/
}

\section{Authors' contributions}

PL, ZJ, CY, ZZ and ZL designed the study. EZ, YZ, SH and LZ collected the data. RT, HC and YH analyzed the data of WES and Sanger sequencing. JZ, HC and $F Z$ performed the IHC staining. PL and ZJ have drafted the work. CY, ZZ and ZL substantively revised it. All authors have read and approved the final manuscript.

\section{Funding}

This work was supported by National Natural Science Foundation of China (82001530, 82171597, 81871215, 81971368, 82171590, 31801219 and 31771650), Clinical Research Innovation Plan of Shanghai General Hospital (KD007-ly01, CTCCR-2019D07), Clinical Research Plan of SHDC (SHDC-

2020CR3077B), the Key Project of Research and Development of Ningxia Hui Autonomous Region of China (2020BFH02002), Shanghai Science and Technology Innovation Action Plan Project (20Y11907600) and Shanghai Sailing Program (20YF1439500 and 20YF1453700).

\section{Availability of data and materials}

The datasets used and analyzed during the current study are available from the corresponding author on reasonable request.

\section{Declarations}

\section{Ethics approval and consent to participate}

This study was approved by the Institutional Ethical Review Committee of Shanghai General Hospital, Shanghai Jiao Tong University (2020SQ199), and the informed consent of clinical data and testicular tissue for research was obtained from the donors. 


\section{Consent for publication}

Not applicable.

\section{Competing interests}

The authors declare that they have no competing interests.

\section{Author details}

'Department of Andrology, Center for Men's Health, Department of ART, Institute of Urology, Urologic Medical Center, Shanghai General Hospital, Shanghai Jiao Tong University School of Medicine, Shanghai 200080, China. ${ }^{2}$ State Key Laboratory of Reproductive Medicine, Nanjing Medical University, Nanjing 211116, China. ${ }^{3}$ The Reproductive Medicine Research Center, The Sixth Affiliated Hospital of Sun Yat-sen University, Guangzhou 510620, China. ${ }^{4}$ School of Life Science and Technology, ShanghaiTech University, Shanghai 201210, China.

Received: 27 October 2021 Accepted: 19 January 2022

Published online: 28 January 2022

\section{References}

1. Tournaye H, Krausz C, Oates RD. Novel concepts in the aetiology of male reproductive impairment. Lancet Diabetes Endocrinol. 2017:5:544-53.

2. de Massy B. Initiation of meiotic recombination: how and where? Conservation and specificities among eukaryotes. Annu Rev Genet. 2013;47:563-99.

3. Gray S, Cohen PE. Control of meiotic crossovers: from double-strand break formation to designation. Annu Rev Genet. 2016;50:175-210.

4. Edelmann W, Cohen PE, Kane M, Lau K, Morrow B, Bennett S, et al. Meiotic pachytene arrest in MLH1-deficient mice. Cell. 1996;85:1125-34.

5. Wang T-F, Kleckner N, Hunter N. Functional specificity of MutL homologs in yeast: evidence for three Mlh1-based heterocomplexes with distinct roles during meiosis in recombination and mismatch correction. Proc Natl Acad Sci. 1999;96:13914-9.

6. Nishant K, Plys AJ, Alani E. A mutation in the putative MLH3 endonuclease domain confers a defect in both mismatch repair and meiosis in Saccharomyces cerevisiae. Genetics. 2008;179:747-55.

7. Svetlanov A, Baudat F, Cohen PE, De Massy B. Distinct functions of MLH3 at recombination hot spots in the mouse. Genetics. 2008;178:1937-45.

8. Manhart CM, Alani E. Roles for mismatch repair family proteins in promoting meiotic crossing over. DNA Repair. 2016;38:84-93.

9. de Vries SS, Baart EB, Dekker M, Siezen A, de Rooij DG, de Boer P, et al. Mouse MutS-like protein Msh5 is required for proper chromosome synapsis in male and female meiosis. Genes Dev. 1999;13:523-31.

10. Kneitz B, Cohen PE, Avdievich E, Zhu L, Kane MF, Hou H, et al. MutS homolog 4 localization to meiotic chromosomes is required for chromosome pairing during meiosis in male and female mice. Genes Dev. 2000;14:1085-97.

11. Edelmann W, Cohen PE, Kneitz B, Winand N, Lia M, Heyer J, et al. Mammalian MutS homologue 5 is required for chromosome pairing in meiosis. Nat Genet. 1999;21:123-7.

12. Akbari A, Padidar K, Salehi N, Mashayekhi M, Almadani N, Sadighi Gilani MA, et al. Rare missense variant in MSH4 associated with primary gonadal failure in both 46, XX and 46, XY individuals. Hum Reprod. 2021;36:1134-45.

13. Tang $D, X u C$, Geng $H$, Gao Y, Cheng H, Ni X, et al. A novel homozygous mutation in the meiotic gene MSH4 leading to male infertility due to non-obstructive azoospermia. Am J Transl Res. 2020;12:8185.

14. Ezeh U, Moore H, Cooke I. A prospective study of multiple needle biopsies versus a single open biopsy for testicular sperm extraction in men with non-obstructive azoospermia. Hum Reprod. 1998;13:3075-80.

15. Hung AJ, King P, Schlegel PN. Uniform testicular maturation arrest: a unique subset of men with nonobstructive azoospermia. J Urol. 2007; 178:608-12.

16. Sha $Y$, Zheng L, Ji Z, Mei L, Ding L, Lin S, et al. A novel TEX11 mutation induces azoospermia: a case report of infertile brothers and literature review. BMC Med Genet. 2018;19:1-7.

17. Yao C, Yang C, Zhao L, Li P, Tian R, Chen H, et al. Bi-allelic SHOC1 loss-offunction mutations cause meiotic arrest and non-obstructive azoospermia. J Med Genet. 2021;58:679-86.
18. Greenbaum MP, Yan W, Wu M-H, Lin Y-N, Agno JE, Sharma M, et al. TEX14 is essential for intercellular bridges and fertility in male mice. Proc Natl Acad Sci. 2006;103:4982-7.

19. Van Der Bijl N, Röpke A, Biswas U, Wöste M, Jessberger R, Kliesch S, et al. Mutations in the stromal antigen 3 (STAG3) gene cause male infertility due to meiotic arrest. Hum Reprod. 2019;34:2112-9.

20. Xiao W-J, He W-B, Zhang Y-X, Meng L-L, Lu G-X, Lin G, et al. In-frame variants in STAG3 gene cause premature ovarian insufficiency. Front Genet. 2019:10:1016.

21. He W-B, Tu C-F, Liu Q, Meng L-L, Yuan S-M, Luo A-X, et al. DMC1 mutation that causes human non-obstructive azoospermia and premature ovarian insufficiency identified by whole-exome sequencing. J Med Genet. 2018;55:198-204.

22. Maor-Sagie E, Cinnamon Y, Yaacov B, Shaag A, Goldsmidt H, Zenvirt S, et al. Deleterious mutation in SYCE1 is associated with non-obstructive azoospermia. J Assist Reprod Genet. 2015;32:887-91.

\section{Publisher's Note}

Springer Nature remains neutral with regard to jurisdictional claims in published maps and institutional affiliations.
Ready to submit your research? Choose BMC and benefit from:

- fast, convenient online submission

- thorough peer review by experienced researchers in your field

- rapid publication on acceptance

- support for research data, including large and complex data types

- gold Open Access which fosters wider collaboration and increased citations

- maximum visibility for your research: over $100 \mathrm{M}$ website views per year

At BMC, research is always in progress.

Learn more biomedcentral.com/submissions 